\title{
Verzeichnis der Mitglieder der Vereinigung der Deutschen Staatsrechtslehrer
}

\author{
Stand vom 15. Dezember 1963
}

\section{Vorstand}

1. K r ü g e r, Dr. Herbert, Professor, Hamburg-Gr. Flottbek, Elbchaussee 184.

2. $\mathrm{K}$ a i s e r, Dr. Joseph H., Professor, Freiburg i. Br., Belfortstraße 11.

3. S c u p in, Dr. Hans Ulrich, Professor, Münster i. W., Robert-Koch-Straße 46.

4. Seidl-Hohenveldern, Dr. Ignaz, Professor, Saarbrücken 3, Hellwigstraße 17.

\section{Mitglieder}

1. A b e nd r ot h, Dr. Wolfgang, Professor, Marburg a. d. Lahn, Wilhelm-Roser-Straße 53.

2. Antoni olli, Dr. Walter, Professor, Wien VII, Mariahilferstraße 22-24/III/II/6.

3. A p e $1 \mathrm{t}$, Dr. Willibalt, Professor, Geh. Regierungsrat, Gräfelfing bei München, Freihamerstraße 32.

4. A r m b r u ster, Dr. Hubert, Professor, Mainz, An der Allee 69.

5. B a a d e, Dr. Hans W., Privatdozent (Kiel), Duke University, School of Law, Durham/USA.

6. B a c h of, Dr. Otto, Professor, Tübingen, Auf dem Kreuz 3.

7. B a d u r a, Dr. Peter, Privatdozent, Erlangen, Hartmannstraße 89.

8. B e c k e r, Dr. jur., Dr. phil. Erich, Professor, Speyer, Philipp-Melanchthon-Straße 10.

9. B e r n h a r d t, Dr. Rudolf, Privatdozent (Heidelberg) Schwetzingen, Hölderlinstraße 5.

10. B e t t e rma n n, Dr. Karl August, Professor, BerlinLichterfelde-West, Baseler Straße 114. 
11. B ö h m e r t, Dr. Viktor, Professor, Kiel, Graf-Spee-Straße 9.

12. B üh le r, Dr. Ottmar, Professor, München 27, Kufsteiner Platz 2/III.

13. B ü l c k, Dr. Hartwig, Professor, Heidelberg, Werrgasse 9.

14. Bulling e r, Dr. Martin, Professor, Freiburg i. Br.Littenweiler, Reinhold-Schneider-Straße 20.

15. C a r s t e n s, Dr. Karl, Professor, Bad Godesberg, Im Meisengarten 25.

16. D a g t o g $1 \circ \mathrm{u}$, Dr. Prodomos, Privatdozent, Heidelberg, Neuenheimer Landstraße 68.

17. D o e h r ing, Dr. Karl, Privatdozent, HeidelbergZiegelhausen, Heinrich-Stoeß-Straße $20 \mathrm{c}$.

18. D r a t $h$, Dr. Martin, Professor, Karlsruhe, Beethovenstraße 9.

19. D ü r i g, Dr. Günter, Professor, Tübingen, Ob dem Viehweidle 12.

20. E h mke, Dr. Horst, Professor, Freiburg i. Br.Littenweiler, Aumattenweg 6.

21. E i c h e n ber ger, Dr. Kurt, Professor, Bern (Schweiz) Universität.

22. E r l e r, Dr. Georg, Professor, Göttingen, Schlegelweg 11.

23. Erma cora, Dr. Felix, Professor, Innsbruck, Universität.

24. Evers, Dr. Hans-Ulrich, Privatdozent, Marburg a.d. Lahn, Ziegelstraße 8.

25. F o r s th of $\mathrm{f}$, Dr. Ernst, Professor, Heidelberg-Schlierbach, Wolfsbrunnensteige 13.

26. Friese $n h a h n$, Dr. Dr. h. c. Ernst, Professor, Bonn, Wegeler Straße 2.

27. Fröhler, Dr. Ludwig, Professor (Nürnberg), Burgthann b. Nürnberg, Am Bahnhof 15.

28. Fromont, Dr. Michel, Professor, Saarbrücken 15, Universität.

29. F u s s, Dr. Ernst-Werner, Privatdozent, Hamburg 20, Im Winkel 3.

30. G e c k, Dr. Wilhelm Karl, Privatdozent, Heidelberg, Heuauerweg 25.

31. G e r b e r, D. Dr. jur. Hans, Professor, Freiburg i. Br., In der Röte 5.

32. Glu m, Dr. Friedrich, Professor, München, Südliche Auffahrtsallee 24. 
33. G ö r g, Dr. Hubert, Professor, Ellersberg, Post Forsbach, Bez. Köln (Marburg a. d. Lahn, Universitätsstraße 10).

34. Grewe, Dr. Wilhelm G., Professor, Botschafter der Bundesrepublik Deutschland bei der NordatlantikpaktOrganisation, Paris, 5, avenue Pierre I er de Serbie.

35. Grundman n, Dr. Siegfried, Professor, Gröbenzell bei München, Puchheimerstraße 30.

36. H a me l, Dr. Walter, Professor, Marburg a.d. Lahn, Rotenberg $1 \mathrm{a}$.

37. H e cke l, Dr. Martin, Professor, Tübingen, Hechingerstraße 33.

38. H e n ke, Dr. Wilhelm, Privatdozent, Geismar, Eisenacher Straße 19.

39. H e r $\mathrm{f}$ a hrdt, Dr. Heinrich, Professor, Marburg a. d. Lahn, Am Weinberg 8.

40. H e s s e, Dr. Konrad, Professor, Freiburg i. Br., Hauptstraße 40.

41. H e t t l a g e, Dr. Karl Maria, Professor, Bad Godesberg, Friedrich-Ebert-Straße 83.

42. F r h r. v. d. H e y d t e, Dr. Friedrich August, Professor, Zellingen a. M., Thomas-Glock-Straße 26.

43. H e y e r, Dr. Friedrich, Professor, Bonn, Humboldtstraße 53.

44. v. H i p p e 1, Dr. Ernst, Professor, Godesberg-Mehlem, Mainzer Straße 119.

45. H o e g n e r, Dr. Wilhelm, Honorarprofessor, Ministerpräsident a. D., München, Am Blumengarten 17.

46. H of $\mathrm{fm}$ a $\mathrm{n} n$, Dr. Gerhard, Professor, Marburg a. d. Lahn, Friedrichstraße 31.

47. $\mathrm{H}$ u b e r, Dr.Ernst Rudolf, Professor, Freiburg-Zähringen, In der Röte 2 (Göttingen, Leonard-Nelson-Straße 19).

48. H u b e r, Dr. Hans, Professor, Muri bei Bern, Mannenriedstraße 5.

49. I m b o d e n, Dr. Max, Professor, Basel, Hirzbodenweg 47.

50. I p s e $\mathrm{n}, \mathrm{Dr}$. Hans Peter, Professor, Hamburg 13, Heinrich-Barth-Straße 25 a.

51. J a c o bi, D. Dr. Erwin, Professor, Leipzig S 3, Meusdorfer Straße 5. 
52. J a hrre is s, Dr. Hermann, Professor, Köln-Klettenberg, Nassestraße 30.

53. J a e n i cke, Dr. Günther, Professor, Heidelberg, Karlsruher Straße 57.

54. J e r u s a l e m, Dr. Franz, Professor, München 27, Flemingstraße 58.

55. K a f k a, Dr. Gustav E., Professor, Wien XIX/117, Franz-Klein-Gasse 1 (Hochschule für Welthandel).

56. K a is e r, Dr. Joseph H., Professor, Freiburg i. Br., Belfortstraße 11.

57. K a ufman n, Dr. jur. Dr. phil. h. c. Erich, Professor, Heidelberg, Schweizerweg 1.

58. K i m m in i ch, Dr. Otto, Professor, Würzburg, Dominikanergasse 8.

59. K i p p, Dr. Heinrich, Professor, Innsbruck, Neue Universität, Innrain 52.

60. Kle in, Dr. Friedrich, Professor, Münster i. W., Prinz-Eugen-Straße 12.

61. K ö t t g e n, Dr. Arnold, Professor, Göttingen, Merkelstraße 43.

62. K ordt, Dr. Erich, Professor (Köln), Düsseldorf, Brehmstraße 53.

63. K r a u s, Dr. Herbert, Professor, Göttingen, Baurat-Gerber-Straße 12.

64. K r ü g e r, Dr. Herbert, Professor, Hamburg-Gr. Flottbek, Elbchaussee 184.

65. K ü $\mathrm{ch}$ e $\mathrm{n} h$ of $\mathrm{f}, \mathrm{Dr}$. Günther, Professor, Würzburg, Seinsheimerstraße 13.

66. L a u n, Dr. jur. Dr. rer. pol. h.c. Rudolf, Professor, Ahrensburg bei Hamburg, Vossberg 2.

67. L e ib holz, Dr. Gerhard, Professor, Karlsruhe, Rheingoldstraße 19.

68. L e is n e r, Dr. Walter, Professor, Erlangen, Kochstraße 2.

69. L e r c h e, Dr. Peter, Professor, Berlin-Dahlem, Am Hirschsprung 15.

70. Li e $r m$ a $n n$, D. Dr. Hans, Professor, Erlangen, Am Meilwald 18. 
71. M a $11 \mathrm{~m}$ an n, Dr. Walter, Professor, Frankfurt a. M., Georg-Voigt-Straße 8.

72. Marti, Dr. Hans, Professor, Bern, Schwanengasse 9.

73. M a u n z, Dr. Theodor, Professor, München-Gräfelfing, Hartnagelstraße 3.

74. M a y e r, Dr. Franz, Professor, Speyer a. Rh., Frhr. v.-Stein-Straße 29.

75. M e der, Dr. Walter, Professor, Berlin-Dahlem, Goßlerstraße 29.

76. Meinzolt, D. Dr. Hans, Honorarprofessor, Staatssekretär a. D., Wessling am See, Oberbayern, Obere Seefeldstraße 16.

77. M e is s n e r, Dr. Boris, Professor, Kiel, Blücherstraße 21.

78. Meli c h a r, Dr. Erwin, Professor, Graz, Juristische Fakultät, Universität.

79. M e n g e r, Dr. Christian-Friedrich, Professor, Kiel, Esmarch-Straße 68.

80. Menzel, Dr. Eberhard, Professor, Kiel, Bülowstraße 16.

81. M e r k, Dr. Wilhelm, Professor, Tübingen, Wildermuthstraße 6.

82. M e r k l, Dr. Adolf Julius, Professor, Wien XIX, Pfarrplatz 1.

83. M e y e r, Dr. Ernst Wilhelm, Professor, Berlin-Wannsee, Bismarckstraße 32.

84. M i r b t, Dr. Hermann, Professor, Berlin-Schlachtensee, Spanische Allee 51.

85. M o s le r, Dr. Hermann, Professor, Heidelberg, Berliner Straße 48.

86. M ün ch, Dr. Fritz, Professor, Heidelberg, Berliner Straße 48.

87. v. $\mathrm{M}$ ü $\mathrm{n} \mathrm{ch}, \mathrm{Dr}$. Ingo, Privatdozent, Frankfurt a. M., Grillparzerstraße 52.

88. N a u m a n n, Dr, Richard, Professor, Lüneburg, Ulzener Straße 40.

89. O b e rm a y er, Dr. Klaus, Professor, Erlangen, Am Röthelheim 58.

90. P a r t s h , Dr. Karl Joseph, Professor, Ingelheim a. Rh., Frankenstraße 10. 
91. P e t e r s, Dr. Dr. h. c. Hans, Professor, Köln-Sülz, Zülpicher Straße 83.

92. P $f$ e if e $r$, Dr. Helfried, Professor, Wien XIII, Lainzer Straße 57.

93. Pirs on, Dr. Dietrich, Professor, Marburg a.d. Lahn, Tischbeinweg 1.

94. $\mathrm{R}$ i d d e r, Dr. Helmut, Professor, Heidebergen über Siegburg, Siebengebirgsstraße 4.

95. R üh l a n d, Dr. Curt, Professor, Braunschweig, Dürerstraße 26.

96. R u m p f, Dr. Helmut, Konsul beim Generalkonsulat der Bundesrepublik Deutschland, Mailand, Via Guerazzi 3.

97. R u p p, Dr. Hans-Heinrich, Privatdozent, Reutlingen, Burgstraße 61.

98. S a l z w e d e l, Dr. Jürgen, Professor, Ippendorf b. Bonn, Gudenauerweg 59.

99. $\mathrm{S} \mathrm{ch} \mathrm{a} \mathrm{ck,} \mathrm{Dr.} \mathrm{Friedrich,} \mathrm{Professor,} \mathrm{Hamburg} \mathrm{13,}$ Nonnenstieg 9/II.

100. Sc h a u m a n n, Dr. Wilfried, Professor, Würzburg, Prümstraße 9.

101. S c h e u n er, Dr. Ulrich, Professor, Bad Godesberg, Beethovenstraße 77.

102. S ch indle r, Dr. Dietrich, Privatdozent, ZollikonZürich, Alte Landstraße 44.

103. S chl o c h a u e r, Dr. Hans Jürgen, Professor, Frankfurt a. M., Mertonstraße 17.

104. S c h m id, Dr. Karl, Professor, Frankfurt a. M., Johann-Klotz-Straße 15.

105. S c h n e id e r, Dr. Hans, Professor, Heidelberg, Ludolf-Krehl-Straße 44.

106. S ch neid e r, Dr. Peter, Professor, Mainz, Goldenluftgasse 2 3/10.

107. S c h n u r, Dr. Roman, Privatdozent, Speyer, Heinrich-Heine-Straße 5.

108. S c h ü le, Dr. Adolf, Professor, Tübingen, Bei der Ochsenweide 17.

109. S chwinge, Dr. Erich, Professor, Marburg a. d. Lahn, von-Harnack-Straße 27.

110. S c u p i n, Dr. Hans Ulrich, Professor, Münster i. W., Robert-Koch-Straße 46. 
111. S e id $1-\mathrm{H}$ oh e n velder n, Dr. Ignaz, Professor, Saarbrücken 3, Hellwigstraße 17.

112. S m e n d, D. Dr. Rudolf, Professor, Göttingen, Am Goldgraben 13.

113. Spanner, Dr. Hans, Professor, München 9 , Candidstraße 24/VII.

114. St e r n, Dr. Klaus, Professor, Berlin 33, Trabener Straße 74.

115. S t ö d t e r, Dr. Rolf, Professor, Hamburg-Altona, Palmaille 49 (Fa. John T. Essberger).

116. Th i e m e, Dr. Werner, Professor, Hamburg-Fuhlsbüttel, Am Karpfenteich 58.

117. U b e r, Dr. Giesbert, Privatdozent, Hamburg 13, Hansastraße 38.

118. Ul e, Dr. Carl Hermann, Professor, Heidelberg, Oberer Gaisbergweg 9.

119. V o g e 1, Dr. Klaus, Privatdozent, Hamburg 13, Böttgerstraße 8 hptr.

120. V o i g t, Dr. Alfred, Professor, Erlangen, Burgbergstr. 53.

121. W a c k e, Dr. Gerhard, Professor, Hamburg-Nienstedten, Ordinger Weg 14.

122. W a 1 t e r, Dr. Robert, Professor, Graz, Universität.

123. W a r n cke, Dr. Friedrich, Privatdozent, Köln-Nippes, Mauenheimer Straße 45.

124. W e b e r, Dr. Werner, Professor, Göttingen, Wackenroder Weg 1.

125. Wehrhahn, Dr. Herbert, Professor, Saarbrücken 15, Universität.

126. W e $\mathrm{n}$ g l e r, Dr. jur. Dr. rer. pol. Wilhelm, Professor, Berlin-Zehlendorf, Werder Straße 15.

127. W enze 1, Dr. Max, Professor, Erlangen, Löhestraße 21.

128. We r te $\mathrm{n}$ b r u ch, Dr. Wilhelm, Privatdozent, Köln, Volksgartenstraße 2.

129. W i n k l e r, Dr. Günther, Professor, Wien 1, Hafnersteig 5/I/7.

130. W o lf f , Dr. Hans J., Professor, Münster i. W., Görresstraße 26.

131. Z a c h e r, Dr. Hans, Professor, Saarbrücken 15, Universität.

132. Z ip peliu s, Dr. Reinhold, Professor, Erlangen, Kochstraße 2. 